\title{
Lumbar facet cyst tuberculosis: a rare cause of cauda equina compression. Illustrative case
}

\author{
Karlo M. Pedro, MD, FAFN, ${ }^{1}$ John Emmanuel R. Torio, MD, ${ }^{1}$ Jonathan P. Rivera, MD, DPSP, ${ }^{2}$ and \\ Ibet Marie Y. Sih, MD, FAFN, FPCS ${ }^{1,3}$

\begin{abstract}
${ }^{1}$ Division of Neurosurgery, Department of Neurosciences, and ${ }^{2}$ Department of Pathology, University of the Philippines-Philippine General Hospital, Manila, Philippines; and ${ }^{3}$ Institute for the Neurosciences, St. Luke's Medical Center, Metro Manila, Philippines
\end{abstract}

\begin{abstract}
BACKGROUND Spinal tuberculosis may present in atypical form to involve only the posterior spinal element with relative sparing of the anterior vertebral body and intervertebral disc. Recognition of this unusual pattern is important to avoid delay in diagnosis and treatment.

OBSERVATIONS The authors report a case of a 59-year-old woman with right-sided radiculopathy and motor weakness. Her lumbosacral magnetic resonance imaging showed a large heterogeneous cyst arising from the right L4-5 facet joint. Laminectomy with excision of the cyst was performed. During surgery, the cyst contained cheese-like material that, on histopathological examination, revealed focal aggregates of tuberculous granuloma. Postoperatively, the patient recovered remarkably with no interval development of instability or any deformity.

LESSONS Facet cyst tuberculosis is rare but should be considered in the differential diagnosis in patients coming from endemic regions. Laminectomy with excision of the cyst along with concurrent antitubercular chemotherapy is a safe and durable treatment option in this case.
\end{abstract}

https://thejns.org/doi/abs/10.3171/CASE20144

KEYWORDS atypical tuberculosis; facet cyst; lumbar synovial cyst; spine tuberculosis

Tuberculosis (TB) is considered the great mimicker, as its clinical and radiological features can imitate a wide range of disease processes in the body. Approximately $10 \%$ of patients with extrapulmonary TB have skeletal involvement, and among them, $50 \%$ will develop spinal TB. ${ }^{1}$ In its typical form, spinal TB is a disease involving two adjacent vertebral bodies with concomitant intervertebral disc destruction and paraspinal soft tissue abscess. ${ }^{2}$ Left untreated, this may invariably lead to extensive bony destruction, deformity, and/or neurological deficits.

An estimated $2 \%-10 \%$ of spinal TB cases present atypically in one of the four forms: (1) involvement only of the posterior element, (2) skip lesion, (3) extradural cord compression without bony involvement, or (4) sacral destruction with pelvic mass. ${ }^{3}$ Atypical spinal TB is often diagnosed on a delayed basis because of its unusual clinical and imaging findings. In endemic regions, prompt recognition of these lesions is important to avoid delay in treatment and prevent possible further progression of neurological deficit. With appropriate therapy, the outcomes of patients with posterior element TB are reported to be equal to those of patients with typical spinal TB. ${ }^{4}$ In this report, we describe a case of an isolated unilateral lumbar facet cyst causing radicular pain that was subsequently proved to be TB.

\section{Illustrative Case}

A 59-year-old woman presented with a 5-month history of progressive low back pain that had suddenly worsened, accompanied by right leg pain and paresthesia. The pain was most severe in the right buttock and lateral leg with radiation to the dorsum of the right foot. She subsequently developed neurological claudication in both legs, limiting her ability to tolerate long-distance walking. She was initially seen at a local hospital, where she was prescribed analgesics, but these offered her no relief. Her physical examination revealed a right $L 4$ motor deficit (4/5) and hypoactive patellar reflex on the right knee. Her sensory examination revealed diminished sensation to light touch and pin prick on the anterior aspect of the right thigh. She did not complain of any bowel or bladder incontinence. Her past medical history was unremarkable. She had no history of cough, weight loss, or fever over the past 3 years.

ABBREVIATIONS CRP = C-reactive protein; ESR = erythrocyte sedimentation rate; MRI = magnetic resonance imaging; $\mathrm{TB}=$ tuberculosis INCLUDE WHEN CITING Published April 12, 2021; DOI: 10.3171/CASE20144.

SUBMITTED December 7, 2020. ACCEPTED January 14, 2021.

(c) 2021 The authors, CC BY-NC-ND 4.0 (http://creativecommons.org/licenses/by-nc-nd/4.0/). 
The results of the patient's laboratory examinations were all normal, except for an elevated erythrocyte sedimentation rate (ESR; $111 \mathrm{~mm} / \mathrm{hr}$; reference range, $0-9 \mathrm{~mm} / \mathrm{hr}$ ) and elevated C-reactive protein (CRP; $29 \mathrm{mg} / \mathrm{dL}$; reference range, $0-4.0 \mathrm{mg} / \mathrm{dL}$ ). The findings of a chest radiograph and an abdominal ultrasound were normal. Lumbosacral radiological images revealed no bony abnormality with stable grade 1 L4-5 listhesis (Fig. 1A). On magnetic resonance imaging (MRI), a large right posterolateral extradural cyst measuring $1.7 \times 1.6 \times 2.8 \mathrm{~cm}$ was seen compressing the thecal sac at the L4-5 level over the right facet joint and was encroaching on the intervertebral foramen. Clumping of nerve roots as a result of thecal sac compression was likewise appreciated (Fig. 1B and C).

The patient underwent L4-5 laminectomy with excision of the facet cyst. The thick-walled cyst contained serous fluid admixed with a friable, cream-colored, solid component firmly attached to the cyst wall. After cyst excision, the thecal sac assumed its midline position, and the exiting L4 nerve root was well decompressed. Postoperatively, the patient's symptoms improved significantly. Her paresthesia and pain resolved completely after surgery. She was able to regain full motor strength of the right leg after 1 month of supplemental physical therapy.

Histopathological examination of the cyst capsule and content revealed several areas of caseous necrosis admixed with fragments of mature bone and cartilage. Clusters of tuberculous granuloma characterized by focal aggregates of histiocytes and lymphocytic infiltration were identified (Fig. 2). Bacteriological examination of the surgical specimen failed to reveal any tuberculous bacilli. The results of TB culture as well as TB polymerase chain reaction assay of the cyst capsule and content were negative. A sputum specimen was submitted for acid-fast bacilli staining and culture, but both results were negative. The patient was started on anti-TB medication and was enrolled in a local health center's directly observed therapy program to ensure compliance with medication. A follow-up radiological
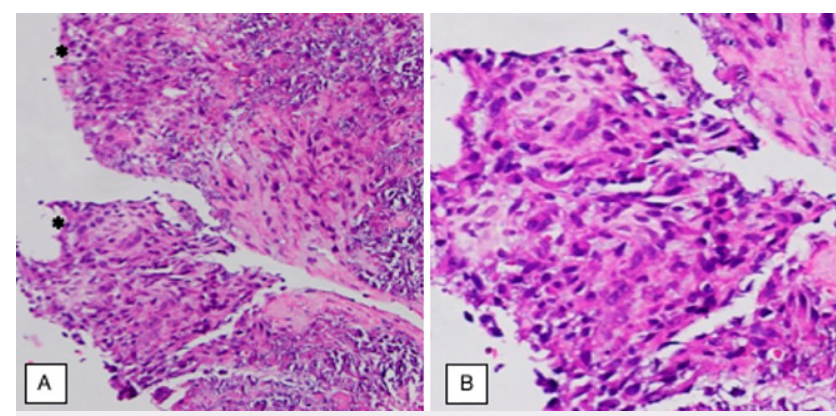

FIG. 2. Histopathological examination. A: Necrotic tissue with dystrophic calcification and granuloma formation (asterisks). Original magnification $\times 100$. B: Higher magnification $(\times 400)$ of granulomas showing aggregates of epithelioid histiocytes with oval nuclei and abundant eosinophilic cytoplasm growing in small clusters and associated with sparse lymphocytes.

examination of the lumbosacral spine 3 months after surgery showed no interval development of sagittal and coronal deformities (Fig. 3). The patient's ESR and CRP levels were also lower postoperatively (ESR, $11 \mathrm{~mm} / \mathrm{hr}$; CRP, $4 \mathrm{mg} / \mathrm{dL}$ ).

\section{Discussion}

\section{Observations}

TB remains a major cause of death and disability worldwide. Despite the discovery of effective and affordable chemotherapy, patients with this chronic granulomatous disease are left with lifelong complications that can significantly affect their quality of life. ${ }^{5}$ Spinal TB is a rare type of mycobacterial infection that can cause back
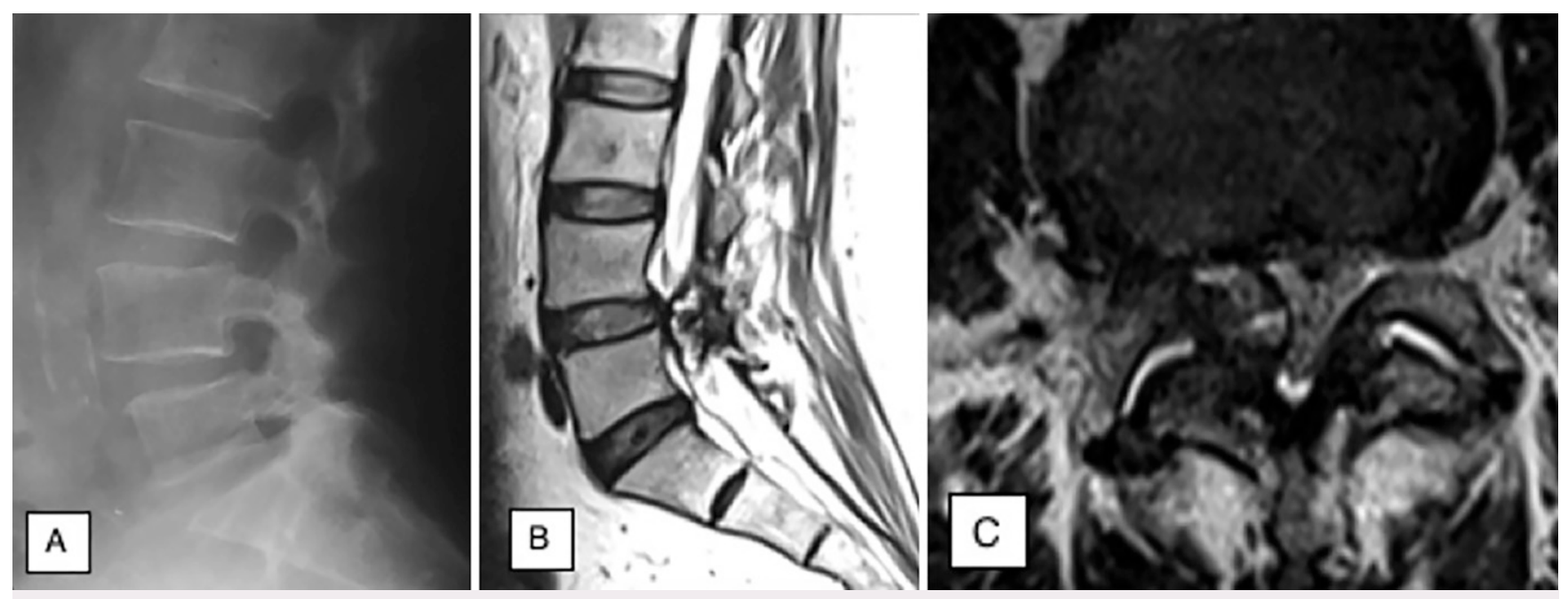

FIG. 1. A: Sagittal radiographic image showing preserved lumbar lordosis with intact anterior and posterior spinal elements and grade $1 \mathrm{~L} 4-5$ listhesis. B: T2-weighted sagittal MRI showing severe spinal stenosis at the L4-5 level due to a large facet cyst demarcated by a hypointense peripheral rim and a hyperintense core. There is mild disc herniation at the L3-4, L4-5, and L5-S1 levels. C: T2-weighted axial MRI demonstrates the degree of thecal sac compression from a right-sided facet cyst causing clumping of the nerve roots at this level. The right intervertebral foramen is almost completely obliterated by the cyst. 


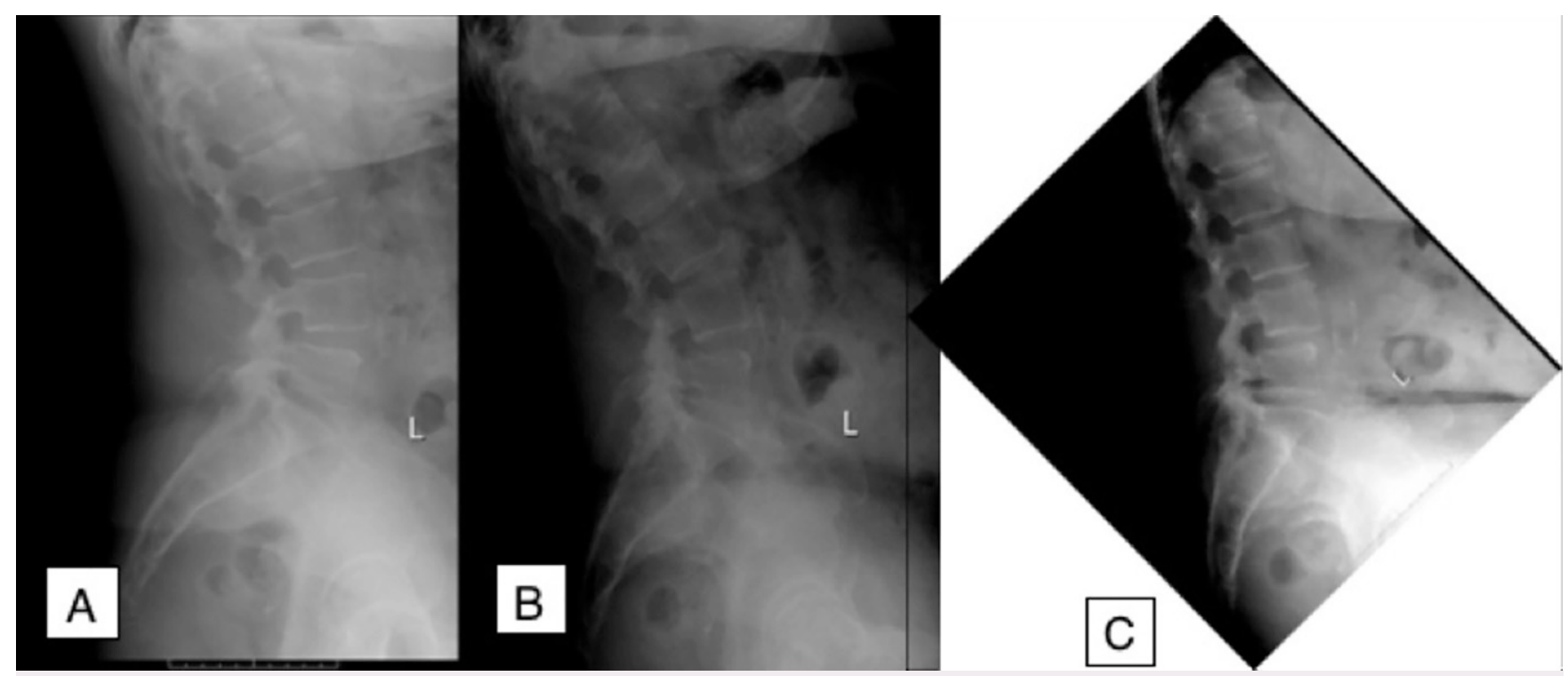

FIG. 3. Follow-up lumbosacral radiographs. A: Sagittal view at 6 months shows no interval development of kyphotic and coronal deformities. Extension (B) and flexion (C) views at the level of the lumbosacral junction show a stable grade 1 spondylolisthesis with intact L4 and L5 bodies.

pain, deformity, and/or neurological deficits. ${ }^{6}$ An estimated $1.7 \%$ of the population worldwide has spinal TB, mostly in countries with a high burden of pulmonary TB. ${ }^{7}$ Although caused by a single agent, Mycobacterium tuberculosis, the clinical pattern of disease has varied presentations. To our knowledge, this is the first report of an isolated tuberculous infection of the lumbar facet presenting as a synovial cyst with no associated instability.

Avadhani et al. ${ }^{8}$ reported a closely related case of a 14-year-old Indian boy with lumbar apophyseal joint TB who presented with back pain and normal neurological findings. On MRI, however, the tuberculous infection involved destruction of not only the L4-5 facet joint but also the ipsilateral pedicle, transverse process, and lamina, with a concomitant soft tissue cold abscess. Despite combined surgery and chemotherapy, the patient developed coronal deformity (scoliosis) requiring additional instrumented fusion. In contrast, in this paper, we describe a patient with only facet joint involvement manifesting as a facet cyst with intact posterior and anterior spinal elements. No instability or deformity was recorded during the course of medical and surgical therapy in this patient.

The pathophysiology of atypical TB remains uncertain. A prevailing hypothesis invokes the role of external venous plexus (Batson) of vertebral veins as the primary pathway of the spread of $M$. tuberculosis from a primary lung or blood focus. ${ }^{9}$ The predominant location of this venous system in the posterior neural arch may explain the occurrence of atypical TB in the facet joint. The lymphatic spread theory and multidrug-resistant TB are two other theories believed to explain the occurrence of pure posterior element TB. ${ }^{10}$

Similar to previously reported literature on spinal $T B$, our patient had no active focus of TB elsewhere in the body at the time of investigation. Agrawal et al. estimated that only $50 \%$ of patients with spinal TB would have an active focus proved by radiological and laboratory examinations at the time of presentation. ${ }^{11}$ Hence, the absence of any extrapulmonary source of TB must not deter a clinician in considering this diagnosis, especially in patients coming from endemic regions.

Early radiological diagnosis of posterior element TB is difficult even in high-burden regions, resulting in delayed diagnosis and even misdiagnosis in some cases. ${ }^{12}$ This is compounded by the fact that spinal TB is characterized by a paucibacillary state, rendering them also difficult to prove by microbiological isolation of the causative agent. ${ }^{6,11}$ As illustrated in this case report, the diagnosis is often based solely on histopathological findings consistent with granulomatous infection, such as the presence of epithelioid granulomas, caseation necrosis, lymphocytic infiltration, and/or presence of Langhans-type giant cells, which is seen in up to $56 \%$ of spinal TB cases. $^{2}$

To date, the management of lumbar facet cysts remains controversial. A thorough review of the literature shows a paucity of guidelines discussing the management of tuberculous facet cysts, and our strategy for this case was patterned on the basis of more numerous studies on degenerative juxtafacet cysts. The question of whether the development of a facet cyst represents a state of spinal instability and hence requires fusion remains a matter of debate. ${ }^{13}$ Proponents of upfront instrumented fusion after laminectomy and cyst excision cite the better rates of excellent or good outcomes than those observed in patients who have undergone decompression surgery alone. ${ }^{14}$ On the contrary, a large retrospective analysis by Lyons et al. ${ }^{15}$ showed no correlation between the extent of laminectomy and/or facetectomy and the development of spinal instability for a lumbar facet cyst. In this case, we elected to perform only a laminectomy and excision of the facet cyst because of the absence of any associated preoperative instability or deformity. Our patient showed significant motor and sensory improvement after surgery, with complete resolution of radicular pain. Interim follow-up evaluation likewise showed no development of deformity or instability postoperatively. Therefore, laminectomy with cyst excision along 
with concurrent anti-TB chemotherapy represents a feasible treatment option in the management of isolated facet joint TB.

\section{Lessons}

Spinal TB can present atypically as an isolated lumbar facet cyst causing radicular pain and weakness. Clinicians must have a high index of suspicion, especially in patients coming from endemic regions, to avoid delay in diagnosis and management. Simple laminectomy with excision of the tuberculous facet cyst is a viable surgical option and does not pose a risk of developing postoperative instability and deformity.

\section{References}

1. Rajasekaran S, Soundararajan DCR, Shetty AP, Kanna RM. Spinal tuberculosis: current concepts. Global Spine J. 2018;8(4 suppl): 96S-108S.

2. Garg RK, Somvanshi DS. Spinal tuberculosis: a review. J Spinal Cord Med. 2011;34(5):440-454.

3. Naim-Ur-Rahman, El-Bakry A, Jamjoom A, et al. Atypical forms of spinal tuberculosis: case report and review of the literature. Surg Neurol. 1999;51(6):602-607.

4. Wang LN, Wang L, Liu LM, et al. Atypical spinal tuberculosis involved noncontiguous multiple segments: case series report with literature review. Medicine (Baltimore). 2017;96(14):e6559.

5. Miller TL, McNabb SJ, Hilsenrath P, et al. Personal and societal health quality lost to tuberculosis. PLoS One. 2009;4(4):e5080.

6. Moon MS. Tuberculosis of spine: current views in diagnosis and management. Asian Spine J. 2014;8(1):97-111.

7. Fuentes Ferrer M, Gutiérrez Torres L, Ayala Ramírez O, et al. Tuberculosis of the spine. A systematic review of case series. Int Orthop. 2012;36(2):221-231.

8. Avadhani A, Shetty AP, Rajasekaran S. Isolated tuberculosis of the lumbar apophyseal joint. Spine J. 2010;10(3):e1-e4.

9. Pande KC, Babhulkar SS. Atypical spinal tuberculosis. Clin Orthop Relat Res. 2002;(398):67-74.
10. Al-Khudairi N, Meir A. Isolated tuberculosis of the posterior spinal elements: case report and discussion of management. JRSM Open. 2014;5(9):2054270414543396.

11. Agrawal V, Patgaonkar PR, Nagariya SP. Tuberculosis of spine. $J$ Craniovertebr Junction Spine. 2010;1(2):74-85.

12. Shim HK, Cho HL, Lee SH. Spinal tuberculosis at the posterior element of spinal column: case report. Clin Neurol Neurosurg. 2014;124:146-150.

13. Boviatsis EJ, Stavrinou LC, Kouyialis AT, et al. Spinal synovial cysts: pathogenesis, diagnosis and surgical treatment in a series of seven cases and literature review. Eur Spine J. 2008;17(6):831837.

14. Khan AM, Synnot K, Cammisa FP, Girardi FP. Lumbar synovial cysts of the spine: an evaluation of surgical outcome. J Spinal Disord Tech. 2005;18(2):127-131.

15. Lyons MK, Atkinson JL, Wharen RE, et al. Surgical evaluation and management of lumbar synovial cysts: the Mayo Clinic experience. J Neurosurg. 2000;93(1 suppl):53-57.

\section{Disclosures}

The authors report no conflict of interest concerning the materials or methods used in this study or the findings specified in this paper.

\section{Author Contributions}

Conception and design: Pedro, Torio, Sih. Acquisition of data: Pedro, Torio, Rivera. Analysis and interpretation of data: all authors. Drafting the article: all authors. Critically revising the article: Pedro, Rivera, Sih. Reviewed submitted version of manuscript: Pedro, Rivera, Sih. Approved the final version of the manuscript on behalf of all authors: Pedro. Study supervision: Sih.

\section{Correspondence}

Karlo M. Pedro: University of the Philippines-Philippine General Hospital, Manila, Philippines. karlopedro_up@yahoo.com. 